PACS 71.25.C, 81.40.V

\title{
A NEW EXPANSION FOR ENERGY SPECTRA OF SOLIDS
}

\author{
Yu.E.Troitskaya, Yu.Yeremeychenkova, E.ZarochentseV \\ Donetsk Physico-Technical Institute \\ Ukrainian National Academy of Sciences \\ 72 R.Luxemburg St., UA-340114 Donetsk-114, Ukraine
}

Received September 12, 1995

\begin{abstract}
A new cluster expansion (CE) is proposed to calculate band structure and adiabatic potential of an insulator at high pressures. This kind of the $\mathrm{CE}$ is developed to permit using Lovdin orthogonalization of atomic orbitals for infinite crystals. The strong binding approach (LCAO method) and OPW one are modified. The technique suggested also fits for the crystals having impuirities, $d$-electrons, etc.
\end{abstract}

\section{Introduction}

The aim of our study is to create the theory allowing one to describe the properties of an insulator at high pressures. The method of calculating energy structure and adiabatic potential of a crystal is proposed in this work.

Available methods of band structure calculation are cumbersome in the case of high pressure and require much machine time. Moreover, we cannot be sure for these methods to give correct results at high compressions. So, at present time we don't know regular ab-initio methods for band structure calculation of insulators under extremal conditions. The aim of the work suggested is to fill in this gap.

For high compressed crystals (or having $d$-electrons, impuirities, etc.) one has to take care for changing electron wave functions of atom in crystal as it is being compressed. In view of the fact, two methods of band structure calculation, namely, the LCAO and OPW are modified by Lovdin orthogonalization and cluster expansion (CE) developed in [7] for the sake of building Bloch functions. The modifications are called CELO and CEOPW respectively (chapters 2 and 3 ). In the chapter 4 of this work new equations for the adiabatic potential (AP) of a crystal are obtained in reciprocal space using $\mathrm{CE}$.

In conventional LCAO method trial Bloch functions are built as for isolated atom orbitals neglecting their overlapping in crystal [1]. In the OPW method, core states are considered by this approach. Beside, there is a number of works [2-5] taking account for the overlapping by the first order in overlapping integrals (OI). However, for the cases mentioned above overlapping among orbitals of neighbour atoms seems to be too large to be treated in those [1-5] ways. Therefore, trial Bloch functions are required to be constructed by exactly orthogonal Wannier functions instead of isolated atom ones. 
The Wannier functions are believed to be approximated by linear combinations of atomic functions orthogonalizated each to others after Lovdin [6]. However, unlike of molecules, in extended solids exact Lovdin orthogonalization is an extremely difficult calculational problem. It is the CE that permits practical work. Using the $\mathrm{CE}$, the problem of Lovdin orthogonalization for localized orbitals of whole crystal is reduced to similar problems for respective clusters. These problems may be solved easily in low orders of the $\mathrm{CE}$ (the $\mathrm{CE}$ for band structure calculation is shown [7] to converge rapidly with $n$ (as a power law in the OI)).

The CE developed by the authors for band structure calculation is analogous to Abarenkov-Antonova's one suggested for electron density of a crystal [8-10] (the CE of [8-10] is used for calculating the AP).

The approach suggested are applicable to calculate the same bands as their "nonorthogonalized" origins but in more wide scale of pressures. They also fit without limitation for the substances having $d$-electrons, impurities, etc.

\section{CELO method}

This method is a modification of the LCAO one. It is disscussed by example of filled bands of insulator. The OI among basis orbitals of the LCAO method may be large as such in the case of high pressures (impuirities, $d$ electrons). Moreover, they are included in LCAO equations being multiplied by the nearest neighbour number $z-10$. So, high-order OI corrections should be taken into account [4]. To permit any further approximation we build, before all, functions $\chi_{\mathbf{l} s}$ exactly (after Lovdin [6]) orthogonalized each to others (we use those as a basis to construct exactly orthogonal set of trial Bloch functions),

$$
\chi_{\mathbf{l} s}=\sum_{\mathbf{l}^{\prime}} \sum_{s^{\prime}} \varphi_{\mathbf{l}^{\prime} s^{\prime}}(\mathbf{r})\left(T^{-1 / 2}\right)_{\mathbf{1}^{\prime} s^{\prime} ; \mathbf{l} s} .
$$

Here $\varphi_{\mathbf{l} s}$ is an isolated atom function, $\mathbf{l}$ is a lattice vector, $N$ is the number of unit cells (the crystals with one atom per cell are considered), $s=(n, l, m)$ denotes occupied states of atom. Each one-particle state (all the quantities are equal $Z / 2, Z$ being the nuclear charge) is supposed to be occupied by two electrons with spins directed "up" and "down". There is no division between core and valence electrons states in this work excluding its last chapter. The orbitals $\varphi_{\mathbf{l} s}$ belonging to the same atom are regarded to satisfy the Shrödinger equation. In equation (1) $T$ is the so-called [8-10] metric matrix with dimension $L \times L(L=N \times Z / 2)$. Its elements are the OI between respective atomic orbitals $\varphi_{\mathbf{l} s}$

$$
T_{\mathbf{l}^{\prime} s^{\prime} ; \mathbf{l} s}=\int \varphi_{\mathbf{I}^{\prime} s^{\prime}}^{*}(\mathbf{r}) \varphi_{\mathbf{l} s}(\mathbf{r}) d^{3} r, \quad \mathbf{l}, \mathbf{l}^{\prime}=1 \ldots N, \quad s, s^{\prime}=1 \ldots Z / 2 .
$$

$T$ is a hermitian matrix. In equation (1) the matrix $T^{1 / 2}$ such that $T^{1 / 2} \cdot T^{-1 / 2}=I, T^{1 / 2} \cdot T^{1 / 2}=T$ and $T^{-1 / 2} \rightarrow I$ as $T \rightarrow I$ is used.

The next step is building a trial Bloch function of CELO method using equation (1)

$$
\Psi_{\mathbf{k} v}(\mathbf{r})=\sum_{s} \psi_{\mathbf{k} s}(\mathbf{r}) C_{s v} \quad \psi_{\mathbf{k} s}(\mathbf{r})=N^{-1 / 2} \sum_{\mathbf{l}} \exp (i \mathbf{k} \mathbf{l}) \chi_{\mathbf{l} s}(\mathbf{r})
$$


with $C_{s v}$ being variational parameters. Index $v$ stands for filled bands. In order that the trial functions equation (3) be orthonormal it is necessary and sufficiently for matrix of coefficients $C_{s v}$ to be unitary.

Altering the equation $E_{\mathbf{k} v}=\left\langle\Psi_{\mathbf{k} v}|H| \Psi_{\mathbf{k} v}\right\rangle /\left\langle\Psi_{\mathbf{k} v}\right| \Psi_{\mathbf{k} v}$ for band energy on the $C_{s v}^{*}$ we obtain the following set of equations for $C_{s v}$

$$
\sum_{s^{\prime}}\left\{\left\langle\psi_{\mathbf{k} s}|H| \psi_{\mathbf{k} s^{\prime}}\right\rangle-E_{\mathbf{k} v} \delta_{s s^{\prime}}\right\} C_{s^{\prime} v}=0
$$

Here $H=T+V(\mathbf{r})$ is the Hamiltonian of an electron, with $V(\mathbf{r})$ including interaction with all nuclei (not with cores) and self-consistent interaction with all the rest electrons in the crystal. Conventionally the $V(\mathbf{r})$ is presented by the superposition $V(\mathbf{r})=\sum_{l} v_{l}$, with $v_{l} \equiv v(|\mathbf{r}-\mathbf{l}|)$ being self-consistent potential centered on the l-th lattice site. The energy $E_{\mathbf{k} v}$ of a band electron is found by solving secular equation for the equation (4) with coefficients $\left\langle\psi_{\mathbf{k} s}|H| \psi_{\mathbf{k} s^{\prime}}\right\rangle$.

To calculate the $\left\langle\psi_{\mathbf{k} s}|H| \psi_{\mathbf{k} s^{\prime}}\right\rangle$ the CE analogous to AbarenkovAntonova's one [8-10] is developed. At first, we bring the $\psi_{\mathbf{k} s}$ in the form suitable for introducing CE. Substituting equation (1) into equation (3) and picking out from $\psi_{\mathbf{k} s}$ its all-OI-neglecting part $\psi_{\mathbf{k} s}^{(0)}$ we obtain

$$
\begin{gathered}
\psi_{\mathbf{k} s}(\mathbf{r})=\psi_{\mathbf{k} s}^{(0)}(\mathbf{r})+\Delta \psi_{\mathbf{k} s}(\mathbf{r}), \quad \psi_{\mathbf{k} s}^{(0)}(\mathbf{r})=N^{-1 / 2} \sum_{\mathbf{l}} \exp (i \mathbf{k} \mathbf{l}) \varphi_{\mathbf{l} s}(\mathbf{r}), \\
\Delta \psi_{\mathbf{k} s}(\mathbf{r})=-N^{-1 / 2} \sum_{\mathbf{l}} \exp (i \mathbf{k} \mathbf{l}) \sum_{\mathbf{l}^{\prime} s^{\prime}} \varphi_{\mathbf{l}^{\prime} s^{\prime}}(\mathbf{r}) \mathcal{P}_{\mathbf{l}^{\prime} s^{\prime} ; \mathbf{l} s} \\
\mathcal{P}=I-T^{-1 / 2} .
\end{gathered}
$$

Basic formula of the $\mathrm{CE}$ for an arbitrary matrix $A_{t}$ corresponding the crystal is [8-10]

$$
A_{t}=\sum_{n=1}^{N} \sum_{[n] \subseteq[N]} Q_{t}[n], \quad Q_{t}[n]=\sum_{m=2}^{n} \sum_{[m] \subseteq[n]}(-1)^{m+n} A_{t}[m] .
$$

Here the $[\eta] \equiv\left[l_{1} \ldots l_{\eta}\right]$ is $\eta$-particle cluster, $[N] \equiv[1 \ldots N]$ being whole the crystal. The second sums in equation (7) goes over all possible different $n$ particle (m-particle) clusters contained in larger one: $[n] \subseteq[N]([m] \subseteq[n])$. The clusters which differ each of other only by transposition of indices in writing $\left[l_{1} \ldots l_{\eta}\right]$ are considered to be equal. Here we need in matrices of $A_{t}=I-T^{-t}$ with $t=1,1 / 2 ; A_{1 / 2}=\mathcal{P}$ equation (6), $A_{1}=P$ equation (15) (see below on):

$$
A_{t}[m]=\left\{\begin{array}{l}
\mathcal{P}[m], \quad t=1 / 2 \\
P[m], \quad t=1
\end{array}=I-(T[m])^{-t}, \quad A_{t}[N] \equiv A_{t} .\right.
$$

All matrices in equation (7) have dimension $L \times L$. The $T[m]$ denotes cluster metric matrix. According to the definition [8-10] it is

$$
T[m]_{\mathbf{l} s ; \mathbf{l}^{\prime} s^{\prime}}=\left\{\begin{array}{l}
T_{\mathbf{l} s ; \mathbf{l}^{\prime} s^{\prime}} ; \mathbf{l} \text { and } \mathbf{l}^{\prime} \in[m] \\
\delta_{\mathbf{l} \mathbf{I}^{\prime}} \delta_{s s^{\prime}} \text { in other cases. }
\end{array}\right.
$$


One can see that each (e.g. $n$-th) order term of the CE is constructed to take account of the overlapping integrals among LO of atoms belonging only to the same $n$-particle cluster. For every $n$ such clusters comprise whole crystal. Beside, each term of the cluster expansion is formed to avoid multifold taking into account the contribution coming from the same cluster. Indeed, the $n$-th order term includes (due to the multiplier $(-1)^{m+n}$ ) contribution of the $n$ - particle cluster minus analogous contributions of the $n-1, n-2$ - particle, etc. clusters contained in this $n$-particle one (these contributions were already accounted in the previous orders of the $\mathrm{CE}$ ).

Although the basic formula of CE (equation (7)) had been obtained in [10] for an arbitrary matrix $A_{t}$, the theorems which permit practical calculations had been proved there only for specific case of matrix $P$ (equation (8) with $t=1)$. The equations for Bloch functions necessary for band structure calculation contain the matrix $\mathcal{P}$ (equation (8) with $t=1 / 2$ ). Therefore, these theorems should be rederived for the $\mathcal{P}$. Here we present only the formulations, with the arguments given in [7].

Theorem I. All elements $Q_{1 / 2}[n]_{\mathbf{l s} ; \mathbf{l}^{\prime} s^{\prime}}$ with $\mathbf{l}$ and(or) $\mathbf{l}^{\prime} \notin[n]$ are equal to zero.

Theorem II. The $Q_{1 / 2}[n]$ is equal to zero even if orbitals of one atom of the cluster $[n]$ are not overlapped with all the rest orbitals of this cluster.

This property allows to develop the nearest (or the second, the third, etc.) neighbours approximation for the $Q_{1 / 2}[n]$.

Theorem III. Extracting square root of cluster matrix $T[m]$ it is enough to solve characteristic equation det $\|\mu I-T[m]\|$ having an order $m \cdot Z / 2$ instead $N \cdot Z / 2$ as for whole metric matrix $T$, with $m$ usually being much smaller than $N$.

Using the CE equation (7), the equation for $\Delta \psi_{\mathbf{k} s}(\mathbf{r})$ from equation (6) becomes

$$
\begin{aligned}
\Delta \psi_{\mathbf{k} s}(\mathbf{r}) & =-\sum_{n=2}^{N} \psi_{\mathbf{k} s}^{n}(\mathbf{r}) \equiv-\sum_{n=2}^{N} \sum_{[n] \subseteq[N]} \psi_{\mathbf{k} s}^{[\mathbf{n}]}(\mathbf{r}), \\
\psi_{\mathbf{k} s}^{[n]}(\mathbf{r}) & =N^{-1 / 2} \sum_{\mathbf{l} \in[n]} \exp (i \mathbf{k} \mathbf{l}) \sum_{\mathbf{l}^{\prime} \in[n], s^{\prime}} \varphi_{\mathbf{l}^{\prime} s^{\prime}}(\mathbf{r}) Q_{1 / 2}[n]_{\mathbf{1}^{\prime} s^{\prime} ; \mathbf{l} s} .
\end{aligned}
$$

The term with $n=1$ in equation (9) is equal to zero because $Q_{1 / 2}[1]=\mathcal{P}[1]$ is the zero matrix. Similarly, the sum over $m$ in the definition of $Q_{1 / 2}[n]$ in equation (6) may be begun from 2.

Using translational symmetry, it may be shown [7] that

$$
\psi_{\mathbf{k} s}^{[n]}(\mathbf{r}+\mathbf{a})=\psi_{\mathbf{k} s}^{[n,-\mathbf{a}]}(\mathbf{r}) \exp (i \mathbf{k a}),
$$

where $[n,-\mathbf{a}] \equiv\left[\mathbf{l}_{1}-\mathbf{a} \ldots \mathbf{l}_{n}-\mathbf{a}\right]$ is a cluster shifted by the lattice vector $\mathbf{a}$ with respect to the original cluster $[n]$. Nevertheless, the function $\psi_{\mathbf{k} s}^{n}(\mathbf{r})$ from equation (9) is a Bloch function i.e. $\psi_{\mathbf{k} s}^{n}(\mathbf{r}+\mathbf{a})=\psi_{\mathbf{k} s}^{n}(\mathbf{r}) \exp (i \mathbf{k a})$.

Substituting the equation (9) into coefficients $\left\langle\psi_{\mathbf{k} s}|H| \psi_{\mathbf{k} s^{\prime}}\right\rangle$ of equation (4) we obtain the CE for them. Solving secular equation for the equation (4) using two-, three-particle, etc. clusters we obtain band structure of a crystal in respective approximation. 


\section{CEOPW method}

Let's go on discussing the CEOPW method as to conduction bands of an insulator. In this method basis plane waves are orthogonalized to wave functions $\Psi_{\mathbf{k} v}(\mathbf{r})$ of filled bands like they are done in ordinary OPW one. A trial Bloch function $\Psi_{\mathbf{k} c}(\mathbf{r})$ of a conduction electron is

$$
\begin{aligned}
\Psi_{\mathbf{k} c}(\mathbf{r}) & =\phi_{\mathbf{k} c}(\mathbf{r})-\sum_{v, \mathbf{k}^{\prime}} \Psi_{\mathbf{k}^{\prime} v}(\mathbf{r}) \int \Psi_{\mathbf{k}^{\prime} v}^{*}\left(\mathbf{r}^{\prime}\right) \phi_{\mathbf{k} c}\left(\mathbf{r}^{\prime}\right) d \mathbf{r}^{\prime}, \\
\phi_{\mathbf{k} c} & =V^{-1 / 2} \sum_{\mathbf{g}} a_{\mathbf{k}}(\mathbf{g}) \exp \{-i(\mathbf{k}+\mathbf{g}) \mathbf{r}\} .
\end{aligned}
$$

Here $a_{\mathbf{k}}(\mathbf{g})$ are variational parameters, $\mathbf{g}$ is reciprocal lattice vector.

The rigid core approximation is used for describing occupied states in conventional OPW. In the CEOPW method the filled band functions $\Psi_{\mathbf{k} v}(\mathbf{r})$ may be orthogonalized each to other as preciously as we desire being calculated by the CELO method. Owing to that the orthogonalization of equation (11) is of the same accuracy. In the conventional OPW method this orthogonality is only in the first order in the OI [1].

Substituting equation (1) into equation (4) and writing

$$
\varphi_{v}\left(\mathbf{r}-\mathbf{l}^{\prime}\right)=\sum_{s} \varphi_{s}\left(\mathbf{r}-\mathbf{l}^{\prime}\right) C_{s v},
$$

we bring the $\Psi_{\mathbf{k} v}$ into the form suitable for further work (see equations $(3),(6))$

$$
\begin{aligned}
& \Psi_{\mathbf{k} v}(\mathbf{r})=N^{-1 / 2} \sum_{\mathbf{l}} \exp (i \mathbf{k} \mathbf{l})\left[\varphi_{v}(\mathbf{r}-\mathbf{l})-\sum_{\mathbf{I}^{\prime} v^{\prime}} \varphi_{v^{\prime}}\left(\mathbf{r}-\mathbf{l}^{\prime}\right) \Pi_{\mathbf{l}^{\prime} v^{\prime} ; \mathbf{l v}}\right], \\
& \Pi_{\mathbf{l}^{\prime} v^{\prime} ; \mathbf{l} v}=\delta_{\mathbf{I I}^{\prime}} \delta_{v v^{\prime}}-\sum_{s s} C_{s^{\prime} v^{\prime}}^{*}\left(T^{-1 / 2}\right)_{\mathbf{l}^{\prime} s^{\prime} ; \mathbf{l} s} C_{s v},
\end{aligned}
$$

with $C_{s v}$ being variational parameters of the CELO method. They are regarded to be already calculated. The functions $\Psi_{\mathbf{k} v}$ in the equation (12) depend on pressure both explicitly (via the OI contained in the $\Pi$ ) and implicitly (via the $C_{s v}$ contained in the $\varphi_{v}$ and $\Pi$ ).

Using equation (12) we obtain for the $a_{\mathbf{k}}(\mathbf{g})$ the following set of equations

$$
\begin{gathered}
\sum_{\mathbf{g}^{\prime}}\left\{H_{\mathbf{g g}^{\prime}}(\mathbf{k})-E_{\mathbf{k} c} \delta_{\mathbf{g g}^{\prime}}\right\} a_{\mathbf{k}}\left(\mathbf{g}^{\prime}\right)=0, \\
H_{\mathbf{g g}^{\prime}}(\mathbf{k})=\frac{\hbar^{2}}{2 m}(\mathbf{k}+\mathbf{g})^{2} \delta_{\mathbf{g g}^{\prime}} \\
+V_{C}\left(\mathbf{g}-\mathbf{g}^{\prime}\right)+V_{e x}\left(\mathbf{k}+\mathbf{g} ; \mathbf{k}+\mathbf{g}^{\prime}\right)+V_{P K}\left(\mathbf{k}+\mathbf{g} ; \mathbf{k}+\mathbf{g}^{\prime}\right) .
\end{gathered}
$$

Here

$$
V_{C}(\mathbf{q})=4 \pi e^{2} /\left(q^{2} \Omega_{0}(\rho(\mathbf{q})-Z)\right)
$$

is the form-factor of the Coulomb interaction of exceeding electron with all the rest ones, $\rho(\mathbf{q})$ is the Fourier transform of their density

$$
\rho(\mathbf{r})=2 \sum_{\mathbf{l} s}\left\{\varphi_{s}^{*}(\mathbf{r}-\mathbf{l}) \varphi_{s}(\mathbf{r}-\mathbf{l})-\sum_{\mathbf{l}^{\prime} s^{\prime}} \varphi_{s}(\mathbf{r}-\mathbf{l}) P_{\mathbf{l} s ; \mathbf{l}^{\prime} s^{\prime}} \varphi_{s^{\prime}}^{*}\left(\mathbf{r}-\mathbf{l}^{\prime}\right)\right\} .
$$


In equation (15) $\Omega_{0}$ is the unit cell volume, sums go over atomic states instead of $v$ because $\rho=\sum_{\mathbf{k} v} \Psi_{\mathbf{k} v} \Psi_{\mathbf{k} v}^{*}=\sum_{\mathbf{k} s} \Psi_{\mathbf{k} s} \Psi_{\mathbf{k} s}^{*}$ since the matrix of CELO variational parameters is unitary.

The term $V_{e x}\left(\mathbf{q} ; \mathbf{q}^{\prime}\right)$ in equation (13) is the form-factor of exchange interaction of exceeding electron with all the rest ones

$$
\begin{aligned}
V_{e x}\left(\mathbf{q} ; \mathbf{q}^{\prime}\right)= & -e^{2} /\left(2 \pi^{2}\right) \sum_{v} \int \frac{d^{3} \kappa}{\kappa^{2}}\left\{I_{v}^{*}(\mathbf{q}-\kappa) I_{v}\left(\mathbf{q}^{\prime}-\kappa\right)\right. \\
& -\left[I_{v}^{*}(\mathbf{q}-\kappa) \sum_{v} I_{v^{\prime}}\left(\mathbf{q}^{\prime}-\kappa\right) \Pi_{v^{\prime} v}^{*}\left(\mathbf{q}^{\prime}-\kappa\right)+\text { H.C. }\right] \\
& \left.+\sum_{v^{\prime} v^{\prime \prime}} I_{v^{\prime}}^{*}(\mathbf{q}-\kappa) I_{v^{\prime \prime}}\left(\mathbf{q}^{\prime}-\kappa\right) \Pi_{v^{\prime} v}(\mathbf{q}-\kappa) \Pi_{v^{\prime \prime} v}^{*}\left(\mathbf{q}^{\prime}-\kappa\right)\right\},
\end{aligned}
$$

the $V_{P K}\left(\mathbf{q} ; \mathbf{q}^{\prime}\right)$ is the form-factor of Phylips-Kleynman potential

$$
\begin{aligned}
V_{P K}\left(\mathbf{q} ; \mathbf{q}^{\prime}\right) & =\sum_{v}\left(E_{\mathbf{k} c}-E_{\mathbf{k} v}\right)\left\{I_{v}^{*}(\mathbf{q}) I_{v}\left(\mathbf{q}^{\prime}\right)\right. \\
& -\left[I_{v}^{*}(\mathbf{q}) \sum_{v^{\prime}} I_{v^{\prime}}\left(\mathbf{q}^{\prime}\right) \Pi_{v^{\prime} v}^{*}\left(\mathbf{q}^{\prime}\right)+\text { H.C. }\right] \\
& \left.+\sum_{v^{\prime} v^{\prime \prime}} I_{v^{\prime}}^{*}(\mathbf{q}) I_{v^{\prime \prime}}\left(\mathbf{q}^{\prime}\right) \Pi_{v^{\prime} v}(\mathbf{q}) \Pi_{v^{\prime \prime} v}^{*}\left(\mathbf{q}^{\prime}\right)\right\} .
\end{aligned}
$$

In equations (16)-(17) $I_{v}(\mathbf{q})$ is the Fourier transform of $\varphi_{v}^{*}(\mathbf{r})$,

$$
\Pi_{v^{\prime} v}(\mathbf{q})=\sum_{\mathbf{l}} \Pi_{\mathbf{l} v^{\prime} ; \mathbf{0} v} \exp (i \mathbf{q} \mathbf{l}) .
$$

It may be proved that $\Pi_{v^{\prime} v}(\mathbf{q})=\Pi_{v v^{\prime}}^{*}(\mathbf{q})$.

The CE for matrix $\Pi_{v^{\prime} v}(\mathbf{k})$ becomes

$$
\Pi_{v^{\prime} v}(\mathbf{k})=\sum_{n=2}^{N} \Pi_{v^{\prime} v}^{(n)}(\mathbf{k}), \quad \Pi_{v^{\prime} v}^{(n)}(\mathbf{k})=\sum_{\left[n_{0}\right] \subseteq[N]} \sum_{\mathbf{l} \in\left[n_{0}\right]} \exp (i \mathbf{k} \mathbf{l}) Q_{1 / 2}\left[n_{0}\right]_{\mathbf{l} v^{\prime} ; 0 v} .
$$

Here the $\left[n_{0}\right] \equiv\left[l_{1} \ldots 0 \ldots l_{n}\right]$ stands for $n$-particle cluster containing zero lattice vector.

Substituting equation (18) into equation (16)-(17) we obtain the CE for exchange and Phylips-Kleynman potentials. The CE for the $\rho(\mathbf{r})$ equation (15) (the $\mathrm{CE}$ for matrix $P$, equation (7) with $t=1$ ) contained in the Coulomb potential $V_{C}\left(\mathbf{g}-\mathbf{g}^{\prime}\right)$ is developed in [8-10].

\section{Adiabatic potential}

Adiabatic potential (AP) is the energy of electrons in crystal, with the ions being fixed in arbitrary positions $\mathbf{R}=\mathbf{l}+\mathbf{u}_{\mathbf{1}}$ close to equilibrium ones $\mathbf{l}$. In the Hartree-Fock (HF) approximation the AP is expressed only via oneelectron density matrix $\rho\left(\mathbf{r} \mid \mathbf{r}^{\prime} ;\{\mathbf{R}\}\right)$ depending on ion coordinates $\{\mathbf{R}\}$ as on parameters. In this approximation the AP consists of electron kinetic energy $T_{e}$, electron-electron Coulomb and exchange interactions, $U_{C}$ and $U_{e x}$ respectively, electron-ion interaction $U_{e i}$, and ion-ion one, $U_{i i}$. In this work the $\rho\left(\mathbf{r} \mid \mathbf{r}^{\prime} ;\{\mathbf{R}\}\right)$ is expressed in terms of $\chi_{s}(\mathbf{r}-\mathbf{R})$. 
Using CE, the equation for AP had been obtained in the [8-10] in $\mathbf{r}-$ space. This result is very useful for the imperfect crystals with impurities chaotically spread in the crystal. For perfect crystals under pressure (or for the cases when impurities form ordered superlattices) it is convenient to work with the $\mathrm{CE}$ in reciprocal space. Expanding the $\rho\left(\mathbf{r} \mid \mathbf{r}^{\prime} ;\{\mathbf{R}\}\right)$ as a Fourier series we express the AP in reciprocal space in the following way

$$
\begin{gathered}
T_{e}=\hbar^{2} /(2 m) \sum_{\mathbf{q}} q^{2} f(\mathbf{q} \mid \mathbf{q}) \\
U_{e x}=-e^{2} /\left(4 N \Omega_{0}\right) \sum_{\mathbf{p q} \kappa} v_{C}(\mathbf{q})|S(\mathbf{p})|^{2} f(\kappa \mid \kappa+\mathbf{p}) f^{*}(\kappa+\mathbf{q} \mid \kappa+\mathbf{p}+\mathbf{q}), \\
U_{e i}=-N Z e^{2} / \Omega_{0} \sum_{\mathbf{k}} v_{C}(\mathbf{k})|S(\mathbf{k})|^{2} \rho_{c}(-\mathbf{k}) F(\mathbf{k}) \\
U_{C}=N Z e^{2} /\left(2 \Omega_{0}\right) \sum_{\mathbf{k}} v_{C}(\mathbf{k})|S(\mathbf{k})|^{2}|F(\mathbf{k})|^{2}
\end{gathered}
$$

Here the

$$
f(\kappa \mid \kappa+\mathbf{p})=2 \sum_{s s^{\prime}} I_{s}^{*}(\kappa) V_{s s^{\prime}}(\kappa) I_{s^{\prime}}(\kappa+\mathbf{p}) ; \quad F(\mathbf{p})=\sum_{\kappa} f(\kappa \mid \kappa+\mathbf{p}),
$$

respectively, are Fourier transforms of the nondiagonal and diagonal elements of electron density matrix of an atom affected by the electrons of all the rest atoms of crystal through the orthogonalization. It may be shown that $f\left(\mathbf{q} \mid \mathbf{q}^{\prime}\right)=f^{*}\left(\mathbf{q}^{\prime} \mid \mathbf{q}\right), F(\mathbf{k})=F^{*}(-\mathbf{k})$. The $\rho_{c}(\mathbf{k})$ is the Fourier transform of core electron density. In the equations (20)-(23)

$$
V_{s s^{\prime}}(\mathbf{q})=\delta_{s s^{\prime}}-P_{s s^{\prime}}(\mathbf{q}) ; \quad P_{s s^{\prime}}(\mathbf{q})=\sum_{\mathbf{h}} P_{\mathbf{h} s ; 0 s^{\prime}} \exp (i \mathbf{q h}),
$$

where $\mathbf{h}=\mathbf{l}+\mathbf{u}_{\mathbf{l}}-\mathbf{u}_{\mathbf{0}}$ is the distance between two atoms, one of them being placed near zero lattice vector ( $\mathbf{u}_{0}$ is its displacement). Using the $\mathrm{CE}$ for matrix $P$ (equation (7) with $t=1$ ) developed in [8-10] we obtain the $\mathrm{CE}$ for $\mathrm{HF}$ part of the $\mathrm{AP}$ in reciprocal space.

Beside HF part (equation (20)-(23)) the AP contains many-electron contributions, e.g. Van-der-Vaals interaction. There is a number of problems, with many-particle effects being dramatic. Specifically, for rare gas crystals the Van-der-Vaals term is a single negative part of the AP providing lattice stability. This term may be obtained, for example, by the second order of perturbation theory in the difference between exact and HF potentials [11]

$$
E^{(2)}=-\sum_{\mathbf{k} \mathbf{k}^{\prime} \kappa \kappa^{\prime} v v^{\prime}} \frac{\left|\left\langle\mathbf{k} v \mathbf{k}^{\prime} v^{\prime}\left|v_{C}(1-\hat{p})\right| \kappa^{\prime} c^{\prime} \kappa c\right\rangle\right|^{2}}{E_{\kappa c}+E_{\kappa^{\prime} c^{\prime}}-E_{\mathbf{k} v}-E_{\mathbf{k}^{\prime} v^{\prime}}} .
$$

Here $v_{C}=e^{2} / r$ is pair the Coulomb interaction potential, $\hat{p}$ is the operator transposing primed and unprimed indices. The indices $v$ and $C$ are used here for valence and conduction bands, $E_{\mathbf{k} v}$ and $E_{\kappa c}$ being respective band energies.

Unlike of [11], the functions $|\mathbf{k} v\rangle$ and $|\kappa c\rangle$ are proposed to be calculated by the CEOPW and CELO methods respectively (instead of strong binding approach). 


\section{Discussion}

So, the technique is developed to permit the calculation of band structure and adiabatic potential in the same approach under high pressures. Beside, the methods suggested are necessary in the case of impurities, $d$-electrons even at normal pressure.

Another application of these methods is phase transitions under pressure, specifically, the insulator-metal transitions in rare gas crystals. However, one delicate moment appears in this theory: The results obtained here are valid only in adiabatic approximation, with the adiabatic parameter $\eta=\hbar \omega_{D} / E_{g}\left(\omega_{D}\right.$ is Debye frequency) containing forbidden gap width $E_{g}$ in the denominator [12]. As the pressure increases this gap decreases and the adiabatic parameter may become larger at the transition point. Then, phonons play an active part in forming band structure. Fortunately, insulator-metal transition pressure is estimated [13] to be nonsensitive to the electron-phonon contributions. Thus, the approach suggested does not lose its validity for finding the transition pressure.

Finally, the question about quantitative expedience of using the theory developed occurs. We discuss the behaviour of matrices $S=T-I, \mathcal{P}$ and $P$ in two-particle cluster approximation [7] and the nearest neighbour one for Ne and Ar. In the Tabl.I and II the largest elements of these matrices are given as a functions of relative compression $\Delta V / V_{0}$ (the matrix $S$ is the first order in the OI of the matrices $2 \mathcal{P}$ and $P$ ). It is seen from the tables that the "cluster" responsible for the overlapping differs of the first order in overlapping integrals insignificantly $(\delta$ and $\Delta \sim 0.01)$ until $\Delta V / V_{0} \sim$ 0.7 (about at this value of compression the transition "insulator-metal" is predicted to occur $[4,5])$. So, the theory for band structure calculation $[2,3]$ and for describing insulator-metal transition $[4,5]$ under pressure seems to be quantitatively precious for $\mathrm{Ne}$ and $\mathrm{Ar}$. In the case of hard elements (for example $\mathrm{Kr}, \mathrm{Xe}$ ) this deviation is larger due to the presence of $d$-electrons. So that the next orders in overlapping integrals are required. In other words, cluster expansion should be used.

Table 1. Ne. The overlapping of the nearest neighbour orbitals.

\begin{tabular}{|c|c|c|c|c|c|c|}
\hline$\Delta V / V_{0}$ & $S_{2 p 2 p}^{(\sigma)}$ & $S_{2 p 2 p}^{(\pi)}$ & $\mathcal{P}_{2 p 2 p}^{(\sigma)}$ & $\delta$ & $P_{2 p 2 p}^{(\sigma)}$ & $\Delta$ \\
\hline 0.0 & 0.087 & 0.014 & 0.043 & 0.00 & 0.087 & 0.00 \\
0.4 & 0.238 & 0.047 & 0.119 & 0.00 & 0.238 & 0.00 \\
0.7 & 0.616 & 0.158 & 0.309 & 0.65 & 0.620 & 0.65 \\
\hline
\end{tabular}

Here $S, \mathcal{P}$ and $P$ are the largest elements $(\times 10)$ of overlapping integrals matrix $\hat{S}$ and matrices $\hat{\mathcal{P}}=1-(1+\hat{S})^{-1 / 2}$ and $\hat{\mathcal{P}}=1-(1+\hat{S})^{-1}, \delta=$ $\frac{\mathcal{P}-S / 2}{\mathcal{P}} \times 100 \%, \Delta=\frac{P-S}{P} \times 100 \%$. 
Table 2. Ar. The overlapping of the nearest neighbour orbitals.

\begin{tabular}{|c|c|c|c|c|c|c|c|c|}
\hline$\Delta V / V_{0}$ & $S_{333 p}^{(\sigma)}$ & $S_{3 p 3 p}^{(\pi)}$ & $S_{3 s 3 p}$ & $S_{3 s 3 s}$ & $\mathcal{P}_{33 p p}^{(\sigma)}$ & $\delta$ & $P_{333 p}^{(\sigma)}$ & $\Delta$ \\
\hline 0.0 & 0.286 & 0.052 & 0.120 & 0.023 & 0.143 & 0.00 & 0.286 & 0.00 \\
0.3 & 0.630 & 0.133 & 0.310 & 0.084 & 0.316 & 0.32 & 0.633 & 0.47 \\
0.7 & 1.300 & 0.346 & 0.778 & 0.285 & 0.662 & 1.81 & 1.337 & 2.77 \\
\hline
\end{tabular}

The definitions are the same as in the table 1.

\section{Acknowledgements}

This investigation is possible partly owing to ISF long-term grants $\mathrm{N} \mathrm{N}$ U9B000 and U9B200.

\section{References}

[1] Ziman J.M. The calculation of Bloch functions. // Phys. Stat. Sol., 1974, vol. 26.

[2] Troitskaya E.P. Conduction bands of compressed crystalline neon. // Solid State Commun., 1984, vol. 52, No 3, p. 321-323.

[3] Zarochentsev E.V., Tolpygo K.B., Troitskaya E.P. Pressure effect on band structure of neon. // Phys. Stat. Sol. (b), 1985, vol. 127, p. 175-185.

[4] Zarochentsev E.V., Troitskaya E.P. Band structure of neon under pressure and the transition into metal state. // Fiz. Tv. Tela, 1985, vol. 27, No 8, p. 2474-2478 (in Russian).

[5] Zarochentsev E.V., Troitskaya E.P. Insulator-metal transition in compressed neon. // Fiz. Tv. Tela, 1988, vol. 30, No 8, p. 2367-2371 (in Russian).

[6] Lovdin P.O. Theoretical investigation into some properties of ionic crystals: Thesis. Uppsala, 1948.

[7] Troitskaya E.P., Yeremeychenkova Yu.V., Zarochentsev E.V. High pressure energy spectra theory of strong-binding states of crystals. // Fiz. Tekhn. Vys. Davl., 1994, vol. 4, No 3-4, p. 7-19 (in Russian).

[8] Abarenkov I.V., Antonova I.M. Interatomic interaction in alkali halides. // Phys. Stat. Sol., 1970, vol. 38, No 2, p. 783-797.

[9] Abarenkov I.V., Antonova I.M. Cluster expansion of density matrix and the energy of ionic crystal in the one-electron approximation. // Fiz. Tv. Tela, 1978, vol. 20, No 2, p. 565-569 (in Russian).

[10] Abarenkov I.V., Antonova I.M., Bar'yakhtar V.G., Bulatov V.L., Zarochentsev E.V. The calculation methods in the theory of solids. The electronic structure of ideal and defect crystals. Kiev, Nauk. Dumka, 1991 (in Ukrainian).

[11] Dorman V.L., Zarochentsev E.V., Troitskaya E.P. Cohesion energy and interatomic potential of rare gas crystals. // Fiz. Tv. Tela, 1981, vol. 23, No 6, p. 1581-1588 (in Russian).

[12] Born M., Huang K. Dynamical theory of crystal lattices. Oxford, Clarendon Press, 1954.

[13] Troitskaya E.P., Yeremeychenkova Yu.V., Zarochentsev E.V. Energy spectra theory of conduction electrons in high compressed crystals. // Fiz. Tekhn. Vys. Davl., vol. 4, No 3-4, p. 20-33. 


\title{
НОВИЙ РОЗКЛАД ДЛЯ СПЕКТРУ ЕНЕРГІЇ
} ТВЕРДИХ ТІЛ

\author{
Ю.Троїтська, Ю.Сремейченкова, Є.Зароченцев
}

Розвинуто новий кластерний розклад для розрахунку зонної структури і адіабатичного потенціалу діелектрика в умовах надвисокого тиску. Цей розклад дає можливість використовувати ортогоналізацію атомних орбіталей по Ловдіну у випадку нескінченного кристалу. Модифіковано підхід міцного зв'язку (метод ЛКАО) та метод ортогоналізованих плоских хвиль. Запропоновані модифікації також можуть бути використані для кристалів, які містять домішки, $d$-електрони та ін. 